\title{
COMMENTARY
}

\section{Importance of national plans for Alzheimer's disease and dementia}

\author{
Marc Wortmann
}

\begin{abstract}
Policy makers have a growing interest in Alzheimer's disease and other dementias, which is seen as the main health and social care challenge of the 21st century. The best way to manage dementia at a country level is by developing national plans, comparable to nationwide management of HIV/AIDS or diabetes. This has been done in a limited number of countries, like Australia, South Korea, France, UK and USA. There are some commonalities in the current plans and we have put those together as a learning experience. The value of these plans can increase when they are monitored and evaluated.
\end{abstract}

\section{Introduction}

Policy makers in health and finance have a growing interest in Alzheimer's disease and other dementias, which is seen as the main health and social care challenge of the 21st century [1]. The best way to manage dementia at a country level is by developing national plans, comparable to nationwide management of HIV/ AIDS or diabetes.

Why is this such a big problem? There is no other disease area where the numbers are going up so rapidly (Figure 1). The number of people with dementia was calculated for 2010 at 36 million and with the increasing life expectancy these numbers are estimated to nearly double to 66 million by 2030 and 115 million by the year 2050 and the cost of the disease was estimated at US $\$ 604$ billion or $1 \%$ of global gross domestic product [2]. By that time people aged over 60 years will outnumber those below 15 years [3]. Therefore, the world needs to prepare for this epidemic. Several countries, including Australia, France, South Korea, UK and USA have already developed national plans and others like Japan, Mexico and Peru are working on it (Figure 2).

Dementia policy is a relatively new area and so far we have little experience on effective measures and hardly any evaluation has been done on these plans. Alzheimer's Disease International, the global federation of national Alzheimer associations, is monitoring the current national plans and working on a learning report that could be useful for other countries that want to make a plan. There are some common elements in many of the current plans and we identified the ten areas that appear in most of them (Box 1).

The French government very recently published a comprehensive evaluation of the French national plan [6]. It notes major achievements in diagnosis, support for people with dementia and their families and in the research area and recommends better integration and collaboration between all healthcare and service providers and calls for continuation into a new plan that will include all neurodegenerative diseases. 

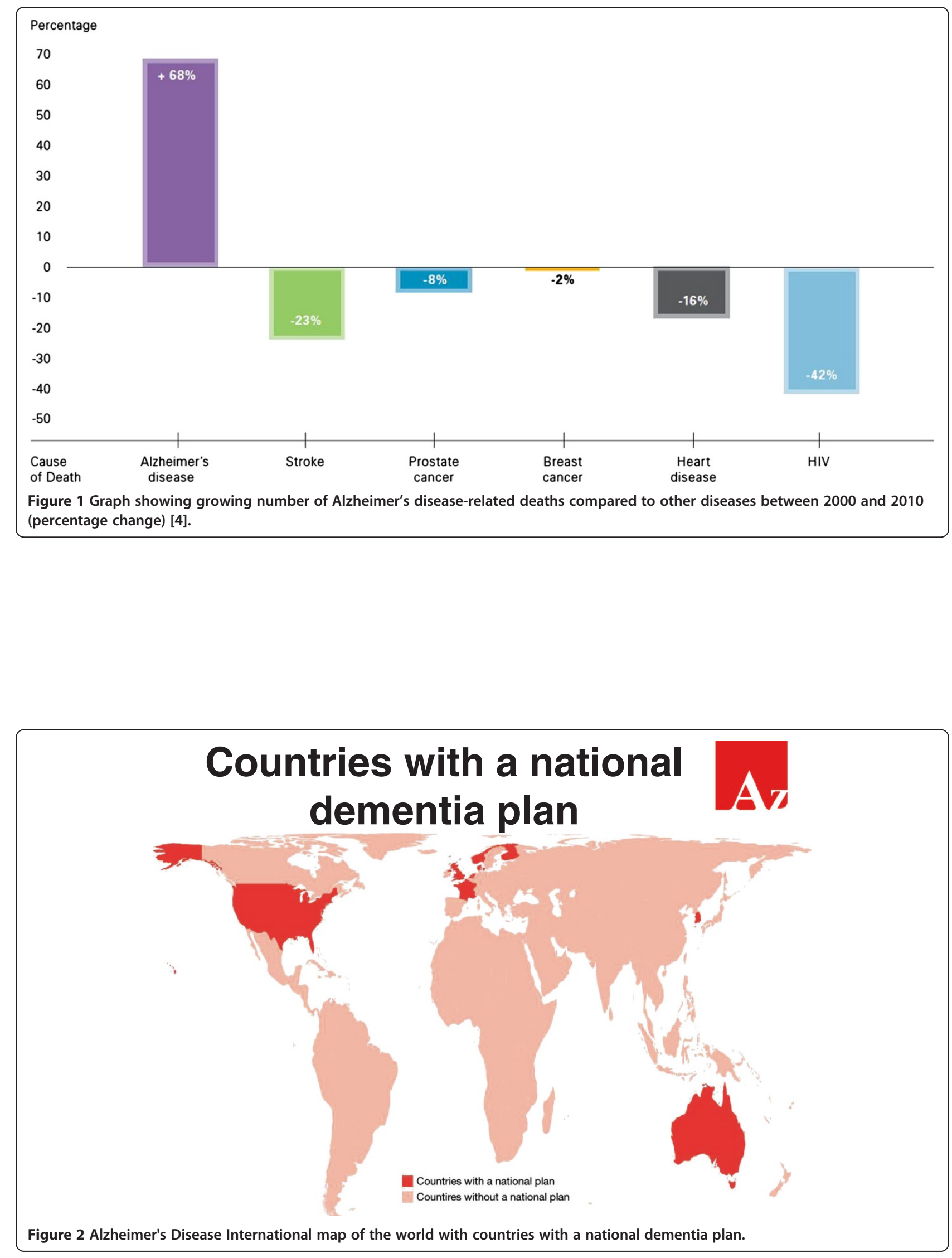


\section{Box 1. Ten common elements in most national dementia plans}

1. Awareness and education

Awareness and education varies from information and educational campaigns to campaigns to eradicate the stigma of dementia, setting up help lines or information websites, organising meetings and monitoring public attitudes and knowledge through population surveys.

A promising concept in this area is the creation of dementia-

friendly communities, which have been developed in several countries in Europe and in Japan. Part of awareness and education are educational programs that aim at a basic understanding of dementia among large groups of the population, which has started in South Korea and Japan and recently was taken over in the UK [5].

\section{2. (Early) diagnosis and treatment}

Actions in this area include establishing memory clinics and other specific services for early diagnosis as well as training of specialists. Regarding treatment, there is specific attention in some countries, like the UK and France, on reducing the use of anti-psychotics and the improvement of correct drug use.

\section{Support at home}

Plans require setting up programs to engage and stimulate people with dementia to take part in programs around rehabilitation and cognitive stimulation and offer more leisure activities to stay active day to day. This should benefit family carers as well.

\section{Family caregiver support}

In connection with support at home, attention is being given to support of family carers through support programs in the local community and creating several types of respite care.

\section{Improve institutional care}

Actions in this area vary across countries, as they all have different health and social care systems. Some are calling for specific units for different patient groups (for instance, people with behavioral problems or younger people with dementia), while others try to transform towards small-scale facilities with more personalised care.

\section{Integrated pathways of care and care coordination}

Integrated pathways of care and care coordination attend to the continuum of care, the explicit focus of the recent Dutch plan and also an important topic in the French Alzheimer Plan. One way to achieve this is to appoint care coordinators or case managers as a single point of contact for families. In the French plan all local stakeholders are encouraged to work together more closely.

\section{Innovative technology}

Attention is also being given to how technology can help assist people at home, through telecare and assisted devices.

\section{Training of healthcare staff}

Training of healthcare staff includes defining competencies for dementia care professionals, continued education for these groups, the development of career pathways and specification by commissioners of necessary dementia training for service providers and hospital staff.

\section{Monitoring}

Some plans pay attention to monitoring and this was well set up in the French plan, as a result of learning from previous plans when they did not monitor well enough. Monitoring can be based on specific indicators in the plan, patient outcomes and care quality indicators.

\section{Research}

Research is gaining more attention in the newer national plans, especially in the USA, but also in the UK Prime Minister's Dementia Challenge [5] and the recently launched new Dutch initiative, which explicitly states that investing in research now could save multiple amounts in the future.

\section{Conclusion}

It is worth doing more research into the results of these national plans and compare them between countries. At the same time we are aware that there is no simple template that can be used everywhere in the world. These policies will only be successful if they adapt to the local situation and involve key stakeholders.

\section{Competing interests}

MW receives a salary from Alzheimer's Disease International.

\section{Acknowledgements}

Prof. Anne Margriet Pot and Dr lonela Petrea are preparing a report with a review on national plans for Alzheimer's Disease International and Bupa. The ten elements are part of that. Expected publication data: October 2013

\section{Published: 06 Sep 2013}

\section{References}

1. Michael H: Why Alzheimer's is Our Children's Nightmare. http://www. huffingtonpost.com/michael-hodin/alzheimers-research-why-alzheimersnightmare_b_3052017.html.

2. World Health Organization, Alzheimer's Disease International: Dementia: a public health priority. http://www.who.int/mental_health/publications/ dementia report_2012/en/.

3. UNFPA, HelpAge International: Ageing in the Twenty-First Century: A Celebration and A Challenge. New York: UNFPA and HelpAge International; 2012:20. 
4. Alzheimer's Association 2013: Alzheimer' Disease Facts \& Figures. www.alz. org/downloads/facts_figures_2013.pdf.

5. Department of Health: The Dementia Challenge. http://dementiachallenge. dh.gov.uk/.

6. Alzheimer Plan 2008>2012. http://www.plan-alzheimer.gouv.fr/remise-durapport-d-evaluation-du.html.

10.1186/alzrt205

Cite this article as: Wortmann: Importance of national plans for Alzheimer's disease and dementia. Alzheimer's Research \& Therapy 2013, 5:40 